\section{Case Reports in Neurology}

Case Rep Neurol 2020;12:70-75

DOI: 10.1159/000507432

Published online: December 14, 2020
(C) 2020 The Author(s)

Published by 2 OPEN www.karger.com/crn

This article is licensed under the Creative Commons Attribution-NonCommercial 4.0 International License (CC BY-NC) (http://www.karger.com/Services/OpenAccessLicense). Usage and distribution for commercial purposes requires written permission.

\title{
Mechanical Thrombectomy and Stenting for Radiation-Induced Carotid Stenosis-Related Stroke: A Case Report
}

\author{
Yu-Chen Cheng ${ }^{a}$ Wei-Cheng Lin ${ }^{b}$ Chia-Nan Lin ${ }^{c}$ Hou-Chang Chiu ${ }^{a}, b$ \\ aDepartment of Neurology, Fu Jen Catholic University Hospital, Fu Jen Catholic University,

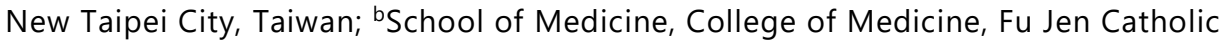 \\ University, New Taipei City, Taiwan; 'Department of Medical Imaging, Fu Jen Catholic \\ University Hospital, Fu Jen Catholic University, New Taipei City, Taiwan
}

\section{Keywords}

Radiation-induced carotid stenosis - Radiotherapy - Acute ischemic stroke - Mechanical thrombectomy $\cdot$ Carotid artery stenting $\cdot$ Carotid artery occlusion

\begin{abstract}
Radiation-induced stenosis of the carotid artery is a significant risk factor for large-vessel ischemic stroke, which usually leads to significant impairment of neurological function. We performed intra-arterial thrombectomy on a 63-year-old male patient who had laryngeal cancer and postradiation carotid stenosis. He presented with acute-onset dysarthria and left hemiplegia. Brain computed tomography perfusion scan showed right middle cerebral artery ischemic change. Angiography confirmed total occlusion of the right internal carotid artery. Intra-arterial mechanical thrombectomy with carotid stenting was performed immediately, and recanalization was achieved. The patient fully recovered and was discharged after a 1-week hospitalization. Our experience suggests that early intervention for radiation-related carotid stenosis might be essential and beneficial for the outcome of large-vessel ischemic stroke.
\end{abstract}




\section{Case Reports in Neurology}

\section{Introduction}

Radiotherapy (RT) is a widely accepted technology for the comprehensive treatment of head and neck cancer [1]. Postradiation vasculopathy is a well-known late-form complication which includes different kinds of vascular injuries, such as small-vessel arteriopathy, Moyamoya disease, vascular malformation, microbleeds, and necrosis [2].

Large-vessel injury is one of the most prominent postradiation vasculopathies. Also, the burden of subsequent cerebrovascular events is more significant among patients with RT for head and neck cancer [3]. Current evidence disclosed that there is an increased risk of carotid stenosis, transient ischemic attack, and general carotid injury among this group of patients.

Ischemic stroke, accounting for around $85 \%$ of all strokes [4], is one of the leading causes of mortality and disability globally [5]. Also, acute management and poststroke care carry tremendous economic burden [6]. Current guidelines recommend recombinant systemic thrombolysis and endovascular mechanical thrombectomy (MT) as a regular management for acute stroke [6]. Moreover, MT has become the gold standard treatment for large-vessel occlusion to restore or improve brain perfusion [6, 7]. Nonetheless, the consensus for MT treatment on radiation vasculopathy-related stroke is still lacking. Here we present a case of acute ischemic stroke in a 63-year-old man with radiation-induced carotid stenosis (RICS) that was successfully treated with MT and carotid artery stenting (CAS).

\section{Case Report}

A 63-year-old man was admitted to the emergency department $3 \mathrm{~h}$ after sudden-onset left hemiparesis. On arrival he had clear consciousness, left central facial palsy, dysarthria, and left hemiplegia. Electrocardiography showed normal sinus rhythm. His National Institutes of Health Stroke Scale (NIHSS) score was 11. He had undergone concurrent chemoradiotherapy for laryngeal cancer 5 years before with no recurrence or notable complications. Otherwise, he had no vascular risk factors, including hypertension, diabetes, or hyperlipidemia. Brain computed tomography perfusion scan (Fig. 1) revealed disproportional hypoperfusion in the right middle cerebral artery (MCA) territory without intracerebral hemorrhage. Besides, computed tomography angiography disclosed tandem occlusive lesions of the right internal carotid artery (ICA) and MCA along with mild to moderate stenosis of the left common carotid artery.

Consequently, we performed endovascular thrombectomy over the M1-M2 segment of the MCA and carotid stenting over the right ICA. Angiography after MT and stenting showed recanalization of the right ICA (Fig. 2). Afterward, he was admitted to the intensive care unit with an NIHSS score of 3. Dual antiplatelet therapy with aspirin and clopidogrel was prescribed. Magnetic resonance imaging revealed acute infarction in the right caudate nucleus and putamen as well as a tiny spot at the right hippocampus. The actual infarction territory was much smaller than the hypoperfusion territory in the perfusion scan. On the day following the procedure, the patient was transferred from the intensive care unit to the ordinary ward. On the day after the transfer, he could walk without assistance in the room, and his NIHSS score remained at 3 . He was discharged after a 1-week rehabilitation course. Two weeks after discharge he had recovered fully without notable neurological deficits. His NIHSS score and modified Rankin score were both 0 . His 90-day modified Rankin score also remained at 0 .

\section{Karger'"}




\section{Case Reports in Neurology}

\section{Discussion}

Cerebrovascular event is one of the most severe delayed complications of RT. The relative risk of transient ischemic attack or stroke could be doubled after head and neck RT [3]. Cranial irradiation could alter the cerebral vascular system, resulting in various vascular complications ranging from microvascular, arterial injuries to large-vessel occlusion [2]. Although the underlying mechanism of radiation-induced vasculopathy is not well known, current evidence suggests that endothelium dysfunction, accelerated atherosclerosis, injury, and occlusion of the vasa vasorum may contribute to the pathophysiology [8]. The radiation-induced endothelial loss could lead to disruption of the blood-brain barrier, thrombi formation, and hemorrhage [2]. Eventually, endothelial proliferation, basement membrane thickening, adventitial fibrosis, and vessel dilation all contribute to vessel remodeling. Also, the radiation damage to the vasculature is dose-dependent. High-dose radiation corresponds to vessels edema, thrombosis, and hemorrhage, while low-dose radiation is related to delayed response of telangiectasia and hemorrhagic infarction [2].

Carotid stenosis is the most prevalent large-vessel disorder among patients who have undergone head and neck RT [9]. The characteristics of RICS are distinct from those of atherosclerotic stenosis in many aspects [10]. Angiographic studies revealed that RICS was mostly located at the common carotid artery and ICA, with more bilateral involvement and more occlusive stenosis. Morphologically, RICS involved longer vascular plaques with deeper and candle-wax ulcerations and maximal stenosis over the end of the plaques. On the contrary, the maximal stenotic sites of atherosclerotic stenosis were close to the center of the whole stenosis. Ultrasonographically, the plaques of RICS tended to be more diffuse and hypoechoic, which could be associated with intraplaque hemorrhage or lipid deposits, even related to the increased risk of ischemic stroke [11]. Histologically, RICS plaques were blended with thickened intima or attached to all layers of vessel walls [10]. Also, RICS demonstrated accelerated atherosclerosis with more necrotic cores, which is caused by the enlargement of intraplaque hemorrhage [8]. The rapid progression of necrotic cores in RICS indicated the vulnerability and mobility of the plaques.

Although the risk of radiation-related stroke is increased among patients with head and neck RT, no trials or data had evaluated the clinical outcome of acute revascularization management in this specific patient group [3]. A previous small-sized trial indicated that the safety and bleeding rate of intravenous thrombolysis treatment were similar between patients with malignancy and control groups [12]. On the other hand, MT is well recognized as a safe and effective technique in general stroke management, especially for large-vessel occlusion [6]. Our case demonstrated that endovascular treatment could be deployed on stroke patients with RICS. We recanalized the main vessels in the brain and thus drastically relieved the neurological deficits. Postthrombectomy angiography revealed residual luminal irregularity $<50 \%$. However, the remaining plaques in the proximal right ICA suggested long-term morphological devastation in the main vessels. Further investigations focusing on the clinical outcome and safety of MT in this selected group are important and necessary.

The preventive effects of medical options, including antiplatelet and vascular risk factors preventive therapy, also remained unclear in patients with RICS [3]. In the American Heart Association/American Stroke Association guideline, CAS is recommended as a class IIa treatment for patients with RICS [13]. However, the restenosis rate after CAS was still higher among the RICS group compared with the atherosclerotic stenosis group ( 43 vs. $13 \%, p<0.05$ ) in a 3-year follow-up study [14]. On the other hand, there are no differences between carotid

\section{Karger'=}




\section{Case Reports in Neurology}

Cheng et al.: Mechanical Thrombectomy and Stenting for Radiation-Induced Carotid Stenosis-Related Stroke: A Case Report

endarterectomy and CAS in patients with RICS. A recent meta-analysis revealed that the risk of periprocedural stroke, mortality, and long-term restenosis rate was similar in these two groups [15].

\section{Conclusion}

Radiation vasculopathy, a common complication of RT for head and neck cancer, may increase the risk of the cerebrovascular events, which usually lead to severe neurological deficits. The current literature provides limited knowledge regarding acute management, including systemic thrombolysis and MT in radiation vasculopathy-related stroke. The successful treatment in our case indicates that MT could be deployed on acute ischemic stroke patients with postradiation ICA occlusion. Such intervention would provide timely restoration of brain perfusion and improve clinical outcome.

\section{Acknowledgement}

We would like to thank the Department of Neuroradiology, Fu Jen Catholic University Hospital, Fu Jen Catholic University for contributing with image scans.

\section{Statement of Ethics}

The authors confirm obtaining written informed consent from the patient for publication of his case, including pictures. The study protocol was approved by the institute's committee on human research.

\section{Conflict of Interest Statement}

The authors have no conflicts of interest to declare.

\section{Funding Sources}

The authors have no funding sources to disclose.

\section{Author Contributions}

Y.-C. Cheng contributed to the diagnosis, physical examination, testing of the patient, and making of the figures. Y.-C. Cheng and W.-C. Lin wrote the manuscript. C.-N. Lin prepared the figures. H.-C. Chiu reviewed and corrected the draft and modified the discussion. All authors read and approved the final manuscript.

\section{Karger'"}




\section{Case Reports in Neurology}

\begin{tabular}{l|l} 
Case Rep Neurol 2020;12:70-75 \\
\hline DOI: 10.1159/000507432 & $\begin{array}{l}\text { (c) 2020 The Author(s). Published by S. Karger AG, Basel } \\
\text { www.karger.com/crn }\end{array}$ \\
\hline
\end{tabular}

Cheng et al.: Mechanical Thrombectomy and Stenting for Radiation-Induced Carotid Stenosis-Related Stroke: A Case Report

\section{References}

1 Cognetti DM, Weber RS, Lai SY. Head and neck cancer: an evolving treatment paradigm. Cancer. 2008 Oct; 113(7 Suppl):1911-32.

2 Murphy ES, Xie H, Merchant TE, Yu JS, Chao ST, Suh JH. Review of cranial radiotherapy-induced vasculopathy. J Neurooncol. 2015 May;122(3):421-9.

3 Plummer C, Henderson RD, O'Sullivan JD, Read SJ. Ischemic stroke and transient ischemic attack after head and neck radiotherapy: a review. Stroke. 2011 Sep;42(9):2410-8.

4 Musuka TD, Wilton SB, Traboulsi M, Hill MD. Diagnosis and management of acute ischemic stroke: speed is critical. CMAJ. 2015 Sep;187(12):887-93.

5 GBD 2016 Neurology Collaborators. Global, regional, and national burden of neurological disorders, 19902016: a systematic analysis for the Global Burden of Disease Study 2016. Lancet Neurol. 2019 May;18(5): 459-80.

6 Powers WJ, Rabinstein AA, Ackerson T, Adeoye OM, Bambakidis NC, Becker K, et al.; American Heart Association Stroke Council. 2018 Guidelines for the Early Management of Patients With Acute Ischemic Stroke: A Guideline for Healthcare Professionals From the American Heart Association/American Stroke Association. Stroke. 2018 Mar;49(3):e46-110.

7 Chen CJ, Ding D, Starke RM, Mehndiratta P, Crowley RW, Liu KC, et al. Endovascular vs medical management of acute ischemic stroke. Neurology. 2015 Dec;85(22):1980-90.

$8 \mathrm{Xu}$ J, Cao Y. Radiation-induced carotid artery stenosis: a comprehensive review of the literature. Intervent Neurol. 2014 Aug;2(4):183-92.

9 Murros KE, Toole JF. The effect of radiation on carotid arteries. A review article. Arch Neurol. 1989 Apr 46(4):449-55.

10 Yu SC, Zou WX, Soo YO, Wang L, Hui JW, Chan AY, et al. Evaluation of carotid angioplasty and stenting for radiation-induced carotid stenosis. Stroke. 2014 May;45(5):1402-7.

11 Lam WW, Liu KH, Leung SF, Wong KS, So NM, Yuen HY, et al. Sonographic characterisation of radiationinduced carotid artery stenosis. Cerebrovasc Dis. 2002;13(3):168-73.

12 Cappellari M, Carletti M, Micheletti N, Tomelleri G, Ajena D, Moretto G, et al. Intravenous alteplase for acute ischemic stroke in patients with current malignant neoplasm. J Neurol Sci. 2013 Feb;325(1-2):100-2.

13 Kernan WN, Ovbiagele B, Black HR, Bravata DM, Chimowitz MI, Ezekowitz MD, et al.; American Heart Association Stroke Council, Council on Cardiovascular and Stroke Nursing, Council on Clinical Cardiology, and Council on Peripheral Vascular Disease. Guidelines for the prevention of stroke in patients with stroke and transient ischemic attack: a guideline for healthcare professionals from the American Heart Association/American Stroke Association. Stroke. 2014 Jul;45(7):2160-236.

14 Protack CD, Bakken AM, Saad WE, Illig KA, Waldman DL, Davies MG. Radiation arteritis: a contraindication to carotid stenting? J Vasc Surg. 2007 Jan;45(1):110-7.

15 Giannopoulos S, Texakalidis P, Jonnalagadda AK, Karasavvidis T, Giannopoulos S, Kokkinidis DG. Revascularization of radiation-induced carotid artery stenosis with carotid endarterectomy vs. carotid artery stenting: a systematic review and meta-analysis. Cardiovasc Revasc Med. 2018 Jul-Aug;19(5 Pt B): 638-44. 


\section{Case Reports in Neurology}

\begin{tabular}{l|l}
\hline Case Rep Neurol 2020;12:70-75 \\
\hline DOI: 10.1159/000507432 & $\begin{array}{l}\text { @ 2020 The Author(s). Published by S. Karger AG, Basel } \\
\text { www.karger.com/crn }\end{array}$ \\
\hline
\end{tabular}

Cheng et al.: Mechanical Thrombectomy and Stenting for Radiation-Induced Carotid Stenosis-Related Stroke: A Case Report
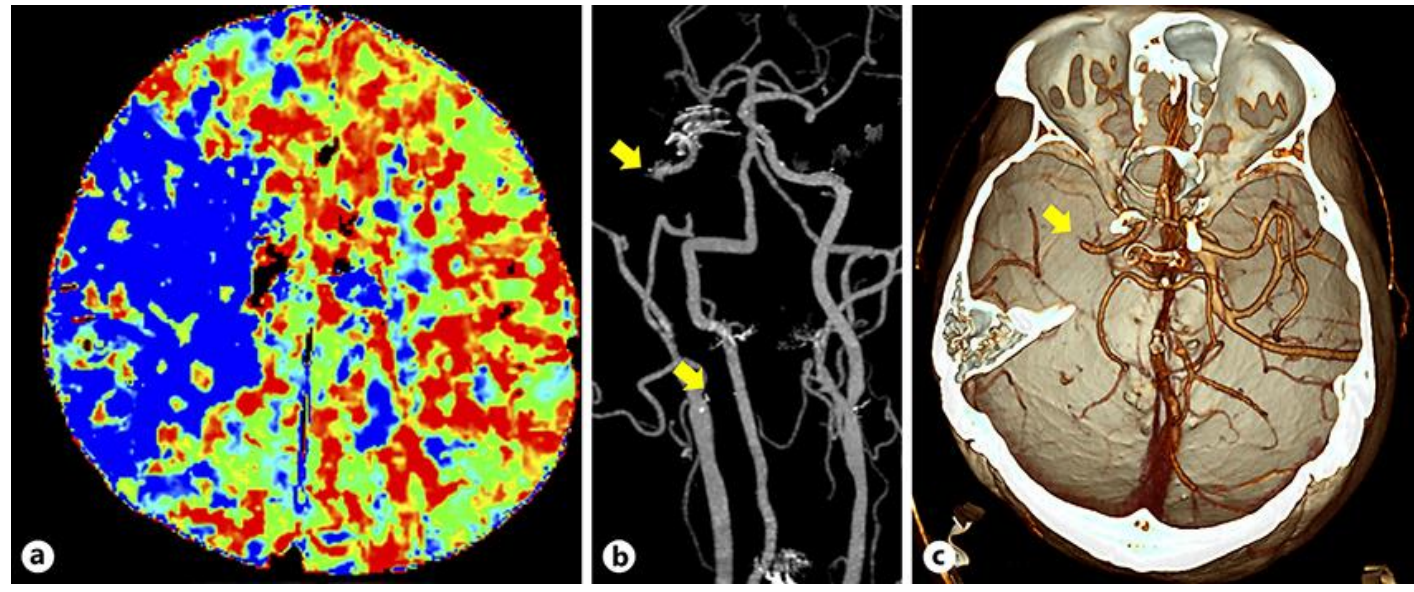

Fig. 1. a Computed tomography perfusion scan before thrombectomy: hypoperfusion in the right MCA territory before thrombectomy. b Computed tomography angiography before thrombectomy: occlusion (arrows) of the right proximal ICA to the horizontal segment of petrous ICA. c Tandem occlusive lesions (arrow) of the MCA. ICA, internal carotid artery; MCA, middle cerebral artery.
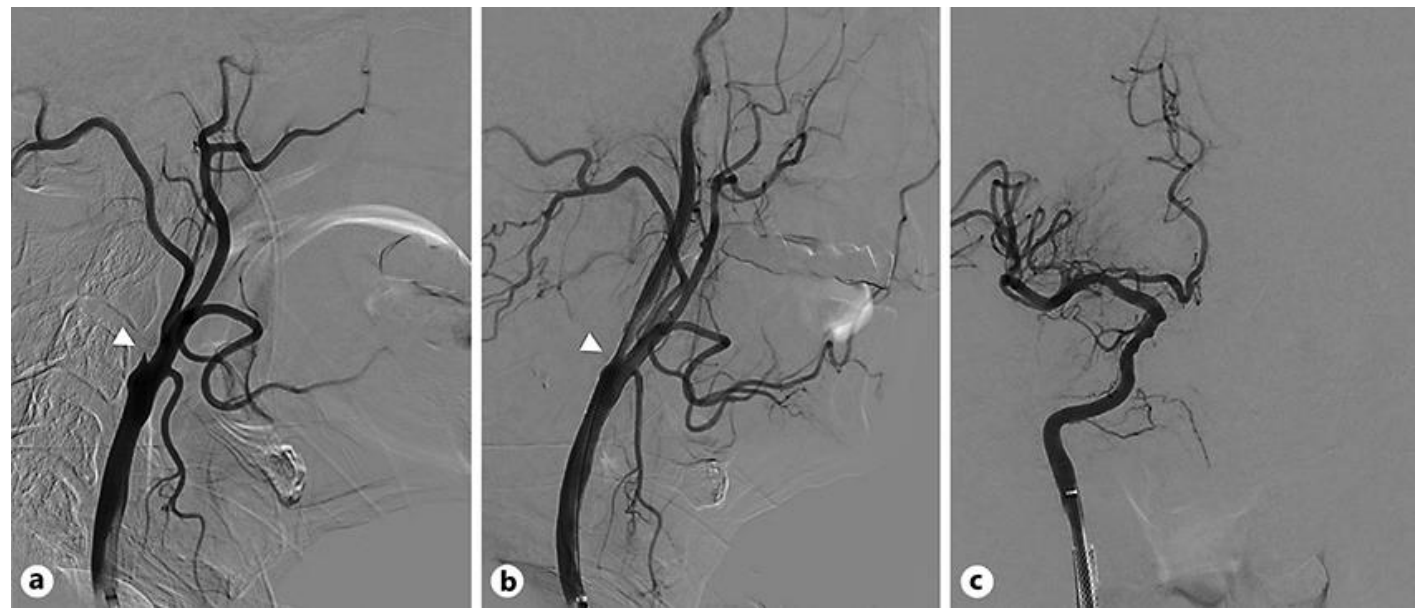

Fig. 2. Cerebral digital subtraction angiography. a Right ICA occlusion. The arrowhead indicates the stump of the right ICA. b Recanalization (arrowhead) of the proximal ICA after MT and stenting. Residual luminal irregularity $<50 \%$. $c$ Recanalization of the occluded right ICA and MCA after MT and stenting. ICA, internal carotid artery; MCA, middle cerebral artery; MT, mechanical thrombectomy. 